\title{
Artificial Disintegration of the Elements. ${ }^{1}$ \\ By Sir ERnest Rutherford, F.R.S.
}

Absorption Curves in Nitrogen and Aluminium.

The variation in the number of scintillations as the absorption in the path of the rays increases from Io $\mathrm{cm}$. is shown in Fig. I. The source of $a$-rays is in

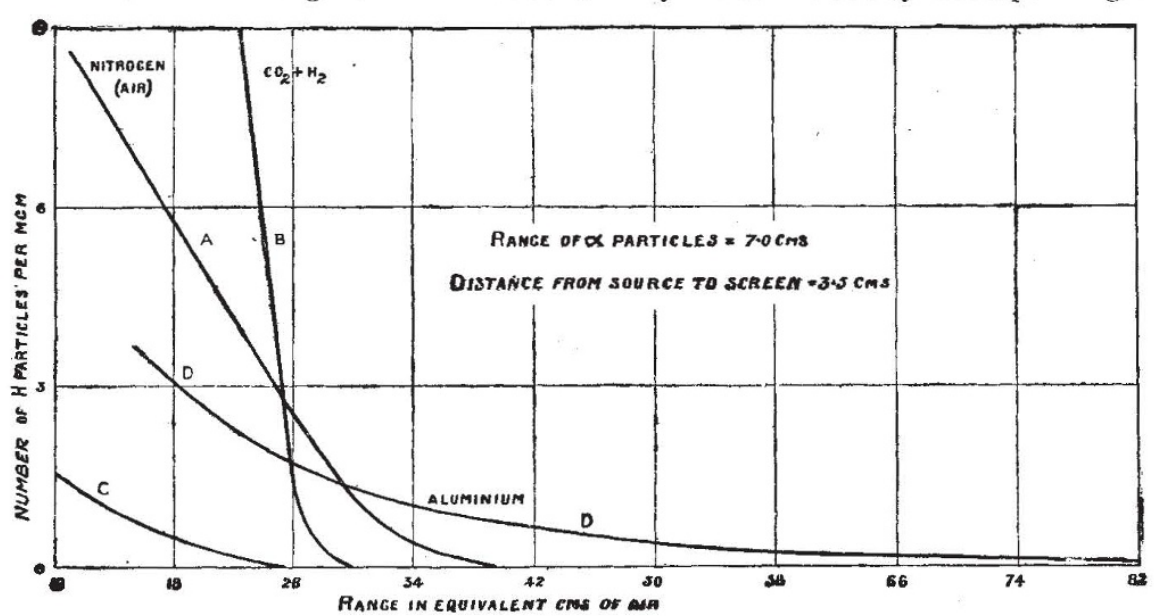

FIG. I.

all cases radium- $C$. Curve $A$ shows the effect in nitrogen (air) where the maximum range is $40 \mathrm{~cm}$. Curve $B$ is the corresponding absorption curve for a mixture of hydrogen and carbon dioxide, about I volume of hydrogen to $\mathrm{I}_{5} 5$ of carbon dioxide, which gives the same stopping power as air for a-rays. The number of scintillations due to hydrogen is very great in this case for absorptions less than $20 \mathrm{~cm}$., but falls off rapidly, and none could be distinguished beyond $30 \mathrm{~cm}$. Curve $C$ gives the natural effect when the air is replaced by dry oxygen. This is small compared with that observed in nitrogen. Curve $D$ shows the effect when an aluminium plate of $3.5 \mathrm{~cm}$. stopping power is placed over the source and the air replaced by oxygen. Thus the particles liberated from aluminium are able to penetrate a much greater thickness than the particles from hydrogen or nitrogen.

It is a matter of great interest to find how the absorption curves for these longrange particles vary with the velocity of the bombarding $a$-particles. This has been examined for two typical elements, nitrogen and aluminium, and the results for the latter are shown in Fig. 2. It was found that to a first approximation the maximum range of the particles liberated from an element was proportional to the range of the bombarding particles. In all cases, the number of scintillations falls off rapidly as the velocity of the $a$-particles is decreased. The effect of velocity is specially marked in aluminium, and few, if any, particles are observed when the

$$
{ }^{1} \text { Continued from p. } 586 .
$$

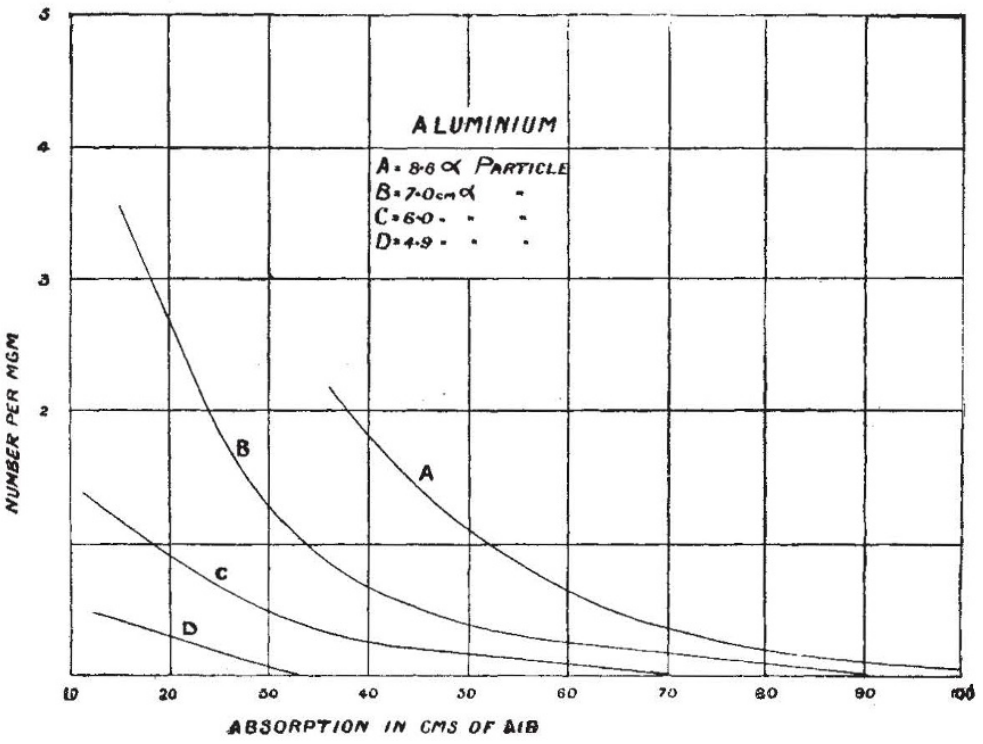

FIG. 2.

rise to long-range particles, to show a similar effect. A possible explanation of this striking result will be given later.

Nature of the Expellen Particles.

It can be shown readily that the long-range particles of an $\alpha$-particle from 7 to $4.9 \mathrm{~cm}$. is only II per cent., we see how rapidly the number falls off with lowering of the velocity. It sems likely no disinthe case of aluminium if the velocity of the $\alpha$-particle falls below a certain critical value. This is not easy to prove conclusively, but, if correct, indicates that the $\alpha$-particle ave a certain critical energy to release an $\mathrm{H}$-atom from the nucleus.

A very striking result was observed in the case of aluminium. It is to be expected that the liberated particles should for the most part be projected in the direction of the bombarding a-particles. Actually, it thent nearly as many were shot in the backward as in the forward direction. No evidence of such an effect was observed in the case of the other elements have not yet been examined from this point of view, but we should expect an element like phosphorus, which gives range of the a-particles is reduced to $4.9 \mathrm{~cm}$. of
air. The effect shown in curve $D$ (Fig. 2) is due almost entirely to the "natural" scintillations from the source. When we remember that the decrease in velocity corresponding with the reduction of the range

NO. 274 I, VOL. IO9] 
liberated from the elements are deflected by a strong magnetic field. By the adoption of special methods, it has been found possible to compare the amount of deflexion of these particles with that shown by the swift $\mathrm{H}$-atoms produced when $\alpha$-particles pass through ordinary hydrogen. It was found that the particles from nitrogen were deflected to about the same extent as the $\mathrm{H}$-particles from hydrogen, and behaved in all respects like swift $\mathrm{H}$-atoms carrying a positive charge. It seemed likely from the first that the corresponding particles from fluorine, phosphorus, and aluminium would also prove to be H-atoms liberated from the nuclei at speeds depending on the nature of the element and the velocity of the impinging particle. This has been confirmed in recent experiments by Dr. Chadwick and myself by a method similar to that employed for nitrogen. The bending of the particles in a magnetic field was determined for an absorption greater than $32 \mathrm{~cm}$. of air, in order that the experiments should not be complicated by the possible presence of hydrogen contamination in the material under examination. The experiments were not easy on account of the small number of particles present under the experimental conditions and it was found necessary to devise a special microscope with a large field of view to carry out the investigation. The experiments were all in accord with the view that the particles from fluorine, phosphorus, and aluminium are swift atoms of hydrogen, and we may conclude that in each case an $\mathrm{H}$-atom is liberated from the nuclei of these elements.

The maximum speed of ejection of the $\mathrm{H}$-atom from the different elements can be estimated approximately by assuming that the law connecting the velocity and range of the $a$-particle holds also for the H-atom, namely, that the velocity is proportional to the cube root of the range. It has been calculated, and also confirmed by experiment, that the maximum speed communicated to a free $\mathrm{H}$-atom by a head-on collision with an $a$-particle of velocity $V$ is $\mathrm{I} \cdot 6 \mathrm{~V}$, while its range in air is about $28 \mathrm{~cm}$. Consequently, the maximum velocity of the H-atom from nitrogen, which has a range $40 \mathrm{~cm}$., is $\mathrm{r} \cdot 8 \mathrm{~V}$, and that from aluminium, with a range of $90 \mathrm{~cm} ., 2 \cdot 37 \mathrm{~V}$. The $a$-particle communicates 0.64 of its energy to a free $\mathrm{H}$-atom in a direct collision, and it can be calculated that all $\mathrm{H}$-atoms which have a range greater than about $56 \mathrm{~cm}$. are projected with energy greater than that of the bombarding $\alpha$-particle. In the case of aluminium, the maximum energy of the $\mathrm{H}$-atom is $\mathrm{I} \cdot 4$ times that of the incident $\alpha$-particle. This is a very interesting result, showing that in some cases there is actually a gain of energy as a result of the disintegration of the aluminium nucleus. We must therefore conclude that at any rate for all collisions in which the liberated $\mathrm{H}$-atom has a range greater than $56 \mathrm{~cm}$. of air, a part of the energy of the $\mathrm{H}$-atom is derived from the disintegrated nucleus. This is analogous in some respects to the well-known gain of energy in the escape of an $a$-particle from a radioactive nucleus.

It must be borne in mind that the amount of disintegration effected by the $\alpha$-particles is on an excessively minute scale. When a particle from radium- $C$ passes through aluminium, it probably passes through the electronic structure of about I00,000 atoms, but only about two $a$-particles in every million get near enough to the inner nucleus to effect the liberation of an H-atom. We know that the collected $\alpha$-particles from I gram of radium give rise to ${ }^{6} \sigma_{3}$ cubic $\mathrm{mm}$. of helium per year. If we suppose that all the $\alpha$-particles from I gram of radium were fired into aluminium, the amount of hydrogen liberated by the disintegration of the aluminium nuclei could not be more than $\mathrm{x} / \mathrm{I} 000$ of a cubic millimetre per year. The amount of hydrogen liberated under possible experimental conditions is thus almost beyond the means of detection by ordinary chemical methods. It has only been possible to study the disintegration by the use of such a delicate method that each $\mathrm{H}$-atom set free produces a visible scintillation on a zinc sulphide screen.

\section{Mechanism of Disintegration.}

From a study of radioactivity, it has been surmised that the $a$-particle or helium nucleus of mass 4 is one of the units of which the atoms are built up. The experiments referred to in this lecture gave the first definite proof that the hydrogen nucleus also is one of the units of the structure of some of the lighter elements. It is of interest to note that $\mathrm{H}$-atoms are only liberated from elements the atomic masses of which are given by $4 n+2$ or $4 n+3$, where $n$ is a whole number. Elements like carbon and oxygen, the atomic masses of which are given by $4 n$, are not affected. This is shown in the following table :-

$\begin{array}{lllll}\text { Element. } & & & \text { Mass. } & 4 n+a . \\ \text { Boron } & \text {. } & \text {. } & \text { II } & 2 \times 4+3 \\ \text { Nitrogen : } & \text {. } & \text {. } & \text { I4 } & 3 \times 4+2 \\ \text { Fluorine : } & \text {. } & \text {. } & \text { I9 } & 4 \times 4+3 \\ \text { Sodium : } & \text {. } & \text {. } & 23 & 5 \times 4+3 \\ \text { Aluminium } & \text {. } & \text {. } & 27 & 6 \times 4+3 \\ \text { Phosphorus } & \text {. } & \text {. } & \text { 3I } & 7 \times 4+3\end{array}$

This result is to be anticipated if the nuclei of these elements are built up of helium nuclei of mass 4 and hydrogen nuclei. In order to account for the liberation of an H-atom from these elements, it is natural to suppose that the $\mathrm{H}$-nuclei are satellites of the main nucleus. If the satellite is not too close to the latter, the $\alpha$-particle in a close collision is able to give the satellite sufficient energy to allow it to escape from the system. It is to be anticipated that the $\mathrm{H}$-satellites are closer to the nucleus in the case of aluminium than in the case of nitrogen, and that consequently more energy is necessary in the case of aluminium to effect their release. It is of interest to note that the chance of liberating a swift $\mathrm{H}$-atom from nitrogen is not more than $\mathbf{r} / 20$ of the chance of setting a free $\mathrm{H}$-atom in corresponding motion. This indicates that it is probably only within certain prescribed limits of velocity of the satellite and position with regard to the central nucleus that the liberation of the satellite is possible.

We have already referred to the fact that the $\mathrm{H}$ atoms from aluminium appear to be released in all directions. Actually, however, the velocity in the backward direction of the a-particles is distinctly less than in the forward direction. Such a result at first suggests that the $\alpha$-particle acts the part of a detonator to the aluminium nucleus and that the energy of the escaping fragments is mainly derived from the nucleus. I think, however, that the following explanation is more probable and in better agreement with experiment.

NO. 274 I, VOL. IO9] 
If we suppose that the $\mathrm{H}$-satellite is describing an orbit round the central nucleus, the direction of escape will depend on the relative position of the $\alpha$-particle and nucleus at the moment of the close collision with the satellite. In the collision shown in
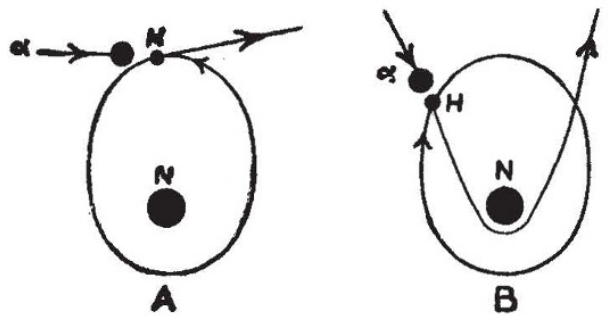

FIG. 3.

Fig. $3, A$, for example, the $\mathrm{H}$-atom will escape in the forward direction of the a-particle; in the collision (Fig. 3, B) the $\mathrm{H}$-atom will describe an orbit round the nucleus and escape in the backward direction. The velocity imparted to the residual nucleus in the forward direction is much greater in the latter than in the former case. Such a view assumes that the forces between the positively charged satellite and nucleus are attractive instead of repulsive very close to the nucleus. This change of sign of the forces seems very probable at short distances from the nucleus, for otherwise it is difficult to understand how a positively charged complex nucleus can hold together.

Another consequence of some interest follows from the possibility of releasing $\mathrm{H}$-atoms from light elements. It is generally supposed, although it is very difficult to obtain direct proof, that the helium nucleus is composed of four hydrogen nuclei and two electrons. In this combination there is a loss of mass, and this is ascribed to the very close combination of the structural units. On modern views of the relation between mass and energy, it follows that the energy liberated in the formation of a helium nucleus is more than three times the energy of the swiftest $\alpha$-particle from radium. We should consequently not expect to be able to break up a helium nucleus with an ordinary a-particle, and this is in agreement with experiment so far as it has gone. In fact, the helium nucleus would appear to be the most stable of all nuclei.

Since, however, in the case of nitrogen, for example, we are able to release an $\mathrm{H}$-atom by means of a slow $\alpha$-particle, it seems clear that the $\mathrm{H}$-satellite is not bound nearly so closely in the nitrogen nucleus as in the case of the helium nucleus. The change of mass due to the emission of energy in binding the $\mathrm{H}$-satellite should consequently be much less than in the case of helium. The mass of the satellite should not differ much from the free $\mathrm{H}$-nucleus of mass $\mathrm{x} \cdot 0077$ in terms of $\mathrm{O}=\mathrm{I} 6$.

If it be supposed that the nitrogen nucleus, for example, is made up of three helium nuclei of total mass 12 and two hydrogen nuclei, the mass of the nitrogen atom should not be exactly $\mathrm{I}_{4} \cdot 00$, but more nearly I4.or. In the case of the light elements, it appears probable that the effective mass of the protons composing the nuclei will vary in different atoms from about $\mathrm{I} \cdot 007$ to $\mathrm{I} \cdot 000$, depending on the closeness of the combination. Consequently, we should expect that the whole number rule found by Aston, which appears to hold for atomic masses to about I in rooo, would be departed from if measurements could be made with still further accuracy.

The next question which arises is whether any other particles beside that of hydrogen can be released by a-ray bombardment. Some time ago, I found that when radium- $C$ was used as a source a small number of bright scintillations were observed, which had a maximum range in air of about $9 \mathrm{~cm}$. It was natural at first to suppose that these were due to a new type of a-rays from the radioactive source. The effect, however, of aluminium screens in reducing the range of these particles led me at first to believe they were generated in the volume of the gases used, namely, nitrogen and oxygen. By comparing the bending of these rays in a magnetic field with that of $\mathrm{H}$-particles from hydrogen, I concluded that they must be atoms of mass about 3 carrying two positive charges. Later experiments have brought home to me the untrustworthiness of this method of fixing the source of the radiation on account of the marked variation in thickness of films of metal foil. Using a more direct and simpler method, I have recently convinced myself that, at any rate in the case of oxygen, the particles have their origin in the radioactive source and not in the volume of the surrounding gas. Under such conditions, the comparative method of estimating the mass of the particles is no longer trustworthy. While a large amount of experiment will be required to fix definitely the nature of the radiation, the general evidence indicates that it consists of particles of mass 4, which are projected from the source and represent a new mode of transformation of radium- $C$.

By the methods outlined we can hope to detect only particles which travel a distance greater than the primary $\alpha$-particles. If, however, a disintegration of an element should occur in which a massive particle is liberated, it is quite probable that the latter may have a range shorter than the $\alpha$-particle. To examine cases of this kind, we can utilise the beautiful method developed by Mr. C. T. R. Wilson for showing the trails of ionising particles. Some experiments of this kind by a modified method have been made by Mr. Shimizu in the Cavendish Laboratory. A number of photographs showing well-marked tracks near the end of the range of the particle have been obtained. Until, however, these photographs are accurately measured up and compared with one another, it is difficult to be certain whether or not these branching tracks can be explained by collisions of the $\alpha$-particle with the nuclei of nitrogen or oxygen. It seems clear, however, that the nuclei involved can travel considerable distances through the gas before being absorbed. If a large number of photographs be taken, it should be possible to settle definitely whether any collisions involving the disruption of atoms occur and to determine the probability of their occurrence. This direct method of attack of the problem, whilst laborious, should give very valuable information on this point.

It appears not improbable that the $\alpha$-particle may occasionally be able to disrupt a helium atom from a complex nucleus like carbon or oxygen, which are believed to be composed of three and four helium nuclei respectively. The fact that the mass of these atoms is nearly an integral multiple of the helium atom suggests 
that the helium nuclei are bound together with much weaker forces than the $\mathrm{H}$-components of the helium nucleus itself. If the structure of the complex nucleus, say, of oxygen is such that the $\alpha$-particle may communicate a considerable fraction of its momentum and energy to a single component, we should expect such disintegration to occur. It is also possible that charged particles of mass about 2 or 3 may exist as secondary building units of the complex nuclei of some elements, but so far no definite evidence of their liberation has been obtained.

I have so far confined my remarks to the disintegrating effects of swift a-rays, but it is important to consider whether the swift $\beta$-rays or energetic $\gamma$-rays from radium are able to produce any effect. We have found that neither $\beta$-nor $\gamma$-rays appear to have the power of giving sufficient energy to a free $\mathrm{H}$-atom in ordinary hydrogen to detect it by the scintillation method, and consequently still less should we expect these rays to liberate a swift $\mathrm{H}$-atom from a complex nucleus. It is possible, however, that these agencies, and particularly $\gamma$-rays of very short wave-length, may be able to liberate an electron and lead in this way to a change of its atomic number. Unless, however, the resulting atom is unstable and breaks up with the emission of a swift particle of the a-ray type, it will be difficult to be certain of such transformations. It should be noted that Slater has shown that $\alpha$-rays are able to excite some very penetrating rays in their passage through ordinary matter. There is some evidence that such high-frequency radiation can only arise from the nuclei of atoms. If this be the case, it may be possible that the $\alpha$-rays in some cases lead to a liberation of a $\beta$-particle from the nucleus and a consequent transformation. This effect, however, must be on a very small scale.

Many attempts have been made in the past to test whether the ordinary atoms can be disintegrated by special agencies. The late Sir William Ramsay, with the characteristic instinct for choosing the best line of attack, made a number of experiments on the effect of the a-rays of radium on matter and concluded that he had obtained evidence of the production of neon from water and the liberation of lithium from copper. These conclusions have not been confirmed by subsequent investigators, and in the light of the experiments described in this lecture it seems very doubtful whether the amount of transformation, even if it occurred, would be sufficient to be detected by ordinary chemical methods such as were employed.

Many instances have been recorded of the appearance of helium in discharge tubes, and it has been suggested that helium is a product of the transformation of the electrodes by the action of the intense electric discharges. The most notable experiments in this direction have been made by Prof. Collie, but the subsequent detailed investigations by Strutt did not confirm his conclusions. It is exceedingly, difficult to prove that the appearance of helium is not due to its occlusion in the electrodes and liberation by the intense heating of the discharge. Similarly, many observations have been made of the steady liberation of hydrogen from electrodes. Winchester, who examined this effect in detail with thin aluminium electrodes, found that hydrogen was released until the electrodes were entirely dissipated. It is very difficult to believe that this hydrogen is a product of the transformation of aluminium when we remember the great energy of the $a$-particle required to effect it. As in the case of helium, it seems more probable that the hydrogen was originally absorbed in the electrodes.

While it is unsafe to be dogmatic on these points, the general evidence indicates that the atoms as a rule are such stable structures and the nuclei are held together by such powerful forces that only a most concentrated source of energy, such as the $\alpha$-particle, is likely to be effective in an attack on such well-protected structures. Even when disintegration takes place, it is on an exceedingly minute scale, and only a few $a$-particles in a million are effective. If we had charged atoms available of ten times the energy of the $a$ particle from radium, we could probably penetrate the nuclear structure of all atoms and occasionally effect their disintegration.

\section{Organisation for Visual Instruction.}

By DR. C. W. Krmmins, Chairman of the Cinema Education Committee.

A BULLETIN on the subject of visual instruction recently received from the University of Wisconsin is of great value as showing the remarkable results which have been obtained by the collection and distribution on an elaborate scale of material for the development of this method of teaching. The fact that in Wisconsin "the circuit plan of distribution" has been in operation for six years "with increasingly gratifying results," enables the authorities to speak with great weight on the subject. The Bulletin deals not only in considerable detail with every department of the organisation, but also gives valuable suggestions for overcoming difficulties such as the transport, storage, and repairing of films and lantern slides. An idea of the great importance attached to visual instruction by the University may be obtained from the fact that a room in the University extension building has been fitted up and equipped with stereopticons, motion- picture machines, and projection apparatus of many of the leading makes. Teachers and committees art invited to visit Madison at any time to inspect thest machines side by side and compare their respective merits with one another. It is also possible at the samt time to become better acquainted with the visual instruction plans and purposes of the university extension division as well as with the stock of material.

In the British Isles the useful part which visual instruction can play in educational methods has long been recognised, as is evidenced by the action of the more important local education authorities in providing the schools with lanterns, lantern slides, pictures, maps, diagrams, botanical and other specimens, and a variety of other material for this purpose. The Lantern Slide Committee of the London County Council has done valuable work for many years in the preparation of a magnificent collection of lantern slides suitable 